\title{
Extraction and purification of biologically active intestinal trefoil factor from human meconium
}

\author{
Kang $\mathrm{Yu}^{1, *}$, Song-Fu Jiang ${ }^{1, *}$, Mao-Fang $\operatorname{Lin}^{2}$, Jian-Bo $\mathrm{Wu}^{1}$ and Jing $\mathrm{Lin}^{3}$ \\ ${ }^{1}$ Department of Hematology, The First Affiliated Hospital of Wenzhou Medical College, China; ${ }^{2}$ Department \\ of Medicine, Medical College of Zhejiang University, China and ${ }^{3}$ Department of Pediatrics, Mount Sinai \\ School of Medicine, New York, USA
}

\begin{abstract}
Intestinal trefoil factor (TFF3/TFF), a member of trefoil factor family (TFF) domain peptides, is normally expressed by goblet cells and secreted into the lumen of the intestinal tract, and plays an important role in the maintenance and repair of the intestinal mucosal barrier. Significant amounts of TFF3/ITF are present in the meconium of human infants. Here, we describe a relatively simple method for extraction and purification of natural human TFF3/ITF from meconium. The purification methods include acid treatment, ammonium sulfate precipitation, isoelectric precipitation, and iron exchange chromatography. A relatively large amount of natural TFF3/ITF can be purified from meconium of full-term infants and this purified, natural TFF3/ITF is biologically active in an ethanol-induced rat gastric mucosal injury model.

Laboratory Investigation (2004) 84, 390-392, advance online publication, 26 January 2004;. doi:10.1038/labinvest.3700042
\end{abstract}

Keywords: intestinal trefoil factor; meconium; newborn infant; gastric mucosa; ethanol

Intestinal trefoil factor (TFF3/ITF), a member of trefoil factor family (TFF) domain peptides, is normally expressed by goblet cells and secreted to the lumen of the intestinal tract. ${ }^{1-3}$ TFF3/ITF plays an important role in the maintenance and repair of the intestinal mucosal barrier. ${ }^{4}$ The distinct threelooped secondary structure contributes to the remarkable resistance of trefoil factors to acid and proteolytic digestion, enables them to function in the harsh environment of the gastrointestinal tract lumen, and allows them to remain biologically active after enteral administration. ${ }^{5,6}$ We have previously demonstrated that significant amounts of TFF3/ITF are present in the meconium of human infants at different gestational ages. ${ }^{7}$ However, whether secreted TFF3/ITF in the human meconium is biologically active remains to be tested. Here, we describe a relatively simple method for the extraction and purification of natural human TFF3/ITF from meconium. The purified natural TFF3/ITF was

Correspondence: J Lin, MD, Jack and Lucy Clark Department of Pediatrics, Division of Newborn Medicine, Mount Sinai School of Medicine, Box 1508, One Gustave L. Levy Place, New York, NY 10029-6574, USA. E-mail: jing.lin@mssm.edu

*Both authors contributed equally to the paper.

Received 17 October 2003; revised 25 November 2003; accepted 04 December 2003; published online 26 January 2004 tested in rats for biological activity by using an in vivo method as described by Babyatsky et al. ${ }^{6}$

Meconium from full-term newborn infants passed within $24 \mathrm{~h}$ of birth was collected and homogenized in $0.1 \mathrm{~N} \mathrm{HCl}$ ( 1 to 3 ratio $\mathrm{w} / \mathrm{v}$ ) with a low-speed homogenizer for $1 \mathrm{~h}$. The homogenates were then centrifuged at $40416 \mathrm{~g}$ at $4^{\circ} \mathrm{C}$ for $1 \mathrm{~h}$ and the supernates were collected for further purification. Preliminary protein precipitation experiments using ammonium sulfate (Gibco BLR, Rockville, MD, USA) at concentrations from 30 to $100 \%(\mathrm{w} / \mathrm{v})$ suggested that TFF3/ITF could not be precipitated by ammonium sulfate up to a concentration of $75 \%$ as indicated by immunoblotting results (data not shown). Therefore, the supernatant fractions were treated with $70 \%$ ammonium sulfate at $4{ }^{\circ} \mathrm{C}$ for $1 \mathrm{~h}$ to precipitate unwanted proteins. After $30 \mathrm{~min}$ of centrifugation at $40416 \mathrm{~g}$, the supernatant fractions underwent isoelectric precipitation with $1 \mathrm{M}$ Tris at $\mathrm{pH} 4.9$ and 4.5, successively. This two-step isoelectric precipitation process was employed because two different isoelectric points for TFF3/ITF have been reported: 4.9 was based on the calculation with protein sequence analysis software-ANTHEPROT (http://antheprot-pbil.ibcp.fr), and 4.5 was reported for recombinant TFF3/ITF. ${ }^{8}$ The precipitate was then dissolved in anion ion exchange chromatography initial buffer (10 mM Tris-HCl, 0.4 M NaCl, pH 8.0) and loaded on a DEAE-Sephadex A25 column 
$\left(1.6 \times 20 \mathrm{~cm}^{2}\right)($ Pharmacia, Uppsala, Sweden), and then eluted by a linear NaCl gradient $(10 \mathrm{mM}$ Tris$\mathrm{HCl}, 0.4-1.3 \mathrm{M} \mathrm{NaCl}, \mathrm{pH}$ 8.0). Two peaks of protein were identified, as determined by absorbance at $A_{280}$. The large second peak corresponded to an estimated molecular size of $6.7 \mathrm{kDa}$ of TFF3/ITF immunoreactivity on Western blotting (Figures 1 and 2a). The final products of the eluted peak were then lyophilized. The purity of the final product was analyzed on a Coomassie-stained SDS-PAGE gel and it was estimated to be over $90 \%$ pure (Figure $2 \mathrm{~b}$ ).

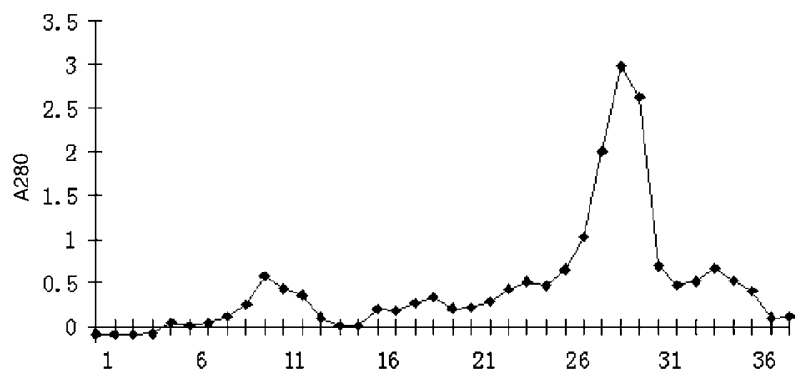

Figure $1 A_{280}$ absorbency of elution fluids from ion exchange chromatography. Two peaks are present, with the large peak II corresponding to an estimated molecular size of $6.7 \mathrm{kDa}$ of TFF3/ ITF on Western blot.

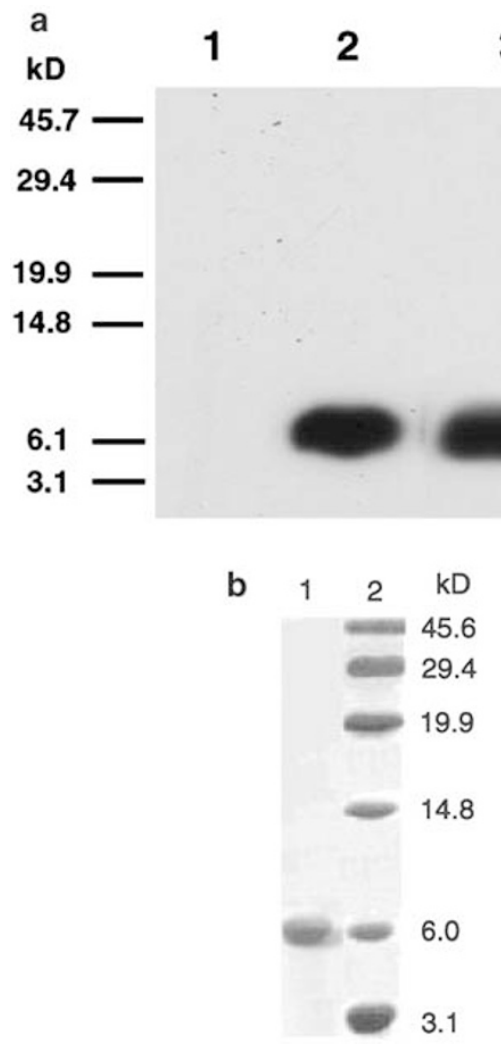

Figure 2 (a) Western blot of final products from peak I (lane 1), peak II (lane 2), positive control of recombinant TFF3/ITF (lane 3), and negative control (lane 4). (b) Coomassie-stained SDS-PAGE gel for analysis of the purity of final product (lane 1). Lane 2 is the molecular weight marker.
Since no reliable in vitro assay is currently available for TFF3/ITF bioactivity, we used an in vivo method as described by Babyatsky et $a l^{6}$ to test whether our purified ITF/TFF3 was biologically active. In all, 24 female Sprague-Dawley rats (weight 180-200g, age 70-80 days) were equally divided into three groups: group 1 as normal/blank control $(n=8)$, group 2 as ethanol gastric injury/ bovine serum albumin (BSA) placebo control $(n=8)$, and group 3 as the ethanol gastric injury/ITF treatment group $(n=8)$. The differences for weight and age among the three groups were not significant $(P>0.05)$. After overnight starvation, rats from group 3 were administered TFF3/ITF $(1 \mathrm{mg} / \mathrm{ml})$ by gastric lavage at a dose of $0.5 \mathrm{ml} / 100 \mathrm{~g}$ body weight, rats from group 2 were administered the same dose of BSA $(1 \mathrm{mg} / \mathrm{ml})$, and rats from group 1 were administrated the same dose of normal saline. After $30 \mathrm{~min}$, gastric mucosal injury was induced in the group 2 and 3 animals via intragastric administration of $90 \%$ ethanol at a dose of $1 \mathrm{ml} / 100 \mathrm{~g}$ of body weight, and group 1 rats were administered the same dose of normal saline as a normal control. After $3 \mathrm{~h}$, rats were killed by $\mathrm{CO}_{2}$ overdose. The stomachs of killed rats were removed and incised along the greater curvature. The stomachs were cleaned, photographed and then the gastric mucosal lesions were scored by two individuals in a blinded manner. The lesions were scored on a $0-10$ scale according to the estimated percent of mucosa covered with lesions (no lesions $=0,100 \%$ mucosal injury $=10$ ) as initially described by Guth et $a .^{9}$ After scoring, the stomachs were fixed in $10 \%$ formaldehyde, embedded in paraffin, and $5-\mu \mathrm{m}$ sections were then stained with $\mathrm{H} \& \mathrm{E}$ for histological examination.

The administration of $90 \%$ ethanol at $1.0 \mathrm{ml} / 100 \mathrm{~g}$ of body weight in group 2 animals induced dilatation of the stomach, submucosal edema, and large areas of submucosal hemorrhage or ulcer. On histological examination, most rats in group 2 presented diverse lesions, including significant mucosal necrosis, disruption of the brush border, capillary dilatation, submucosal edema with large areas of submucosal hemorrhages, and inflammatory cell infiltration. Intragastric administration of purified natural human ITF/TFF3 significantly prevented/ameliorated the ethanol-induced acute gastric mucosal injury (Figure 3). The injury scores on gross examination in group 3 animals were significantly less than those from group 2 $(P<0.001$, ANOVA, Figure 4). These results are similar to the findings of Babyatsky that show the prophylactic use of recombinant TFF3/ITF protects against ethanol-induced gastric injury in rats. These results prove that our purified natural human TFF3/ ITF from meconium is biologically active.

In summary, natural TFF3/ITF present in meconium from full-term infants has the known property of mucosal protection and therefore is biologically active. A relatively large amount of natural TFF3/ITF can be purified from meconium. The 
392
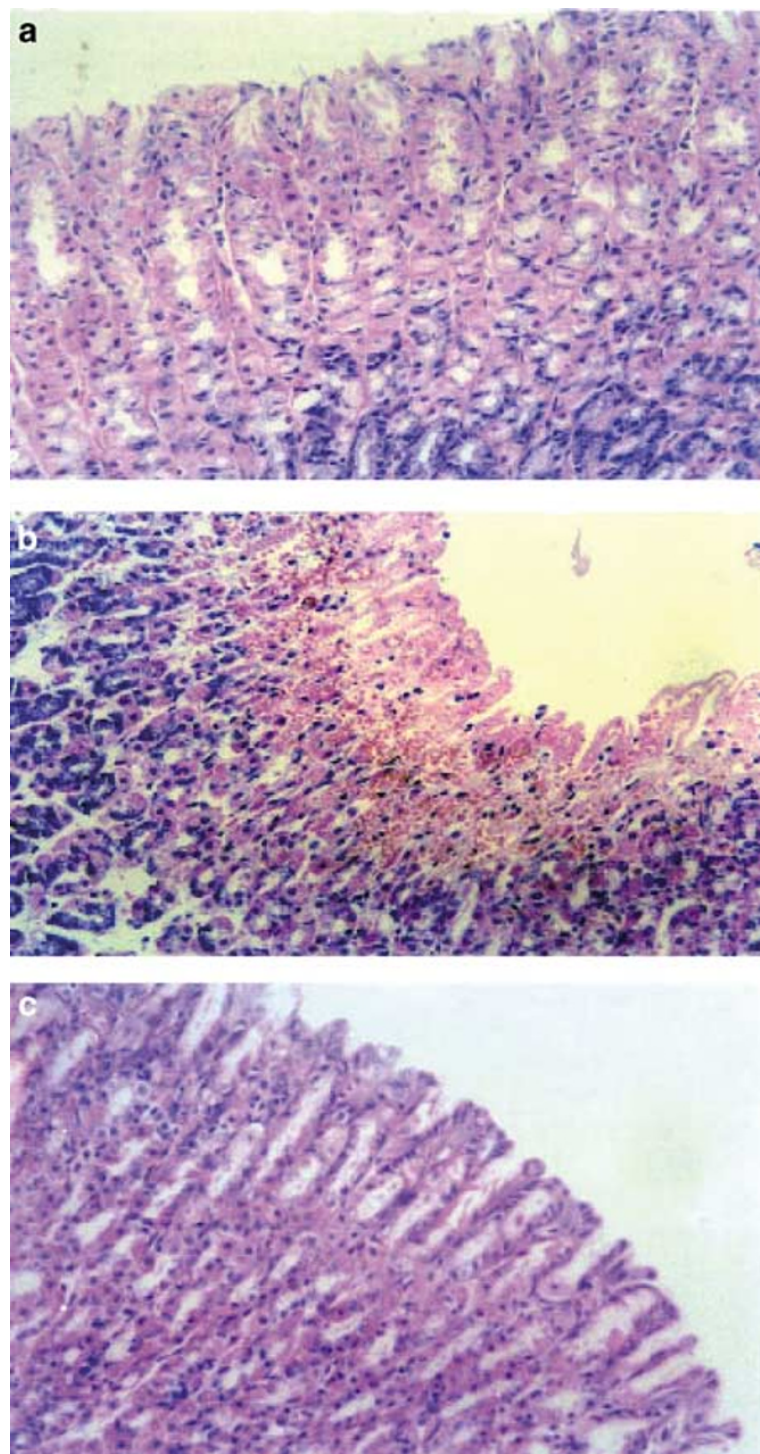

Figure 3 Histology of stomach mucosa from group 1 (a), group 2 (b), and group 3 (c). Mucosal necrosis, submucosal hemorrhage, and inflammatory cell infiltration are present in group 2 but not in group $3(\times 200)$

purification methods including acid treatment, ammonium sulfate precipitation, isoelectric precipitation, and iron exchange chromatography are relatively simple and can be performed in most laboratories. The availability of biologically active natural human TFF3/ITF may facilitate the studies of the biological functions of TFF3/ITF and its underlying mechanisms.

\section{Acknowledgement}

This work was supported by NICHD Grant 1-K0801223 to Dr Jing Lin. We thank Dr Ian Holzman for reviewing the manuscript. The TFF3/ITF-specific antiserum and recombinant TFF3/ITF were kindly

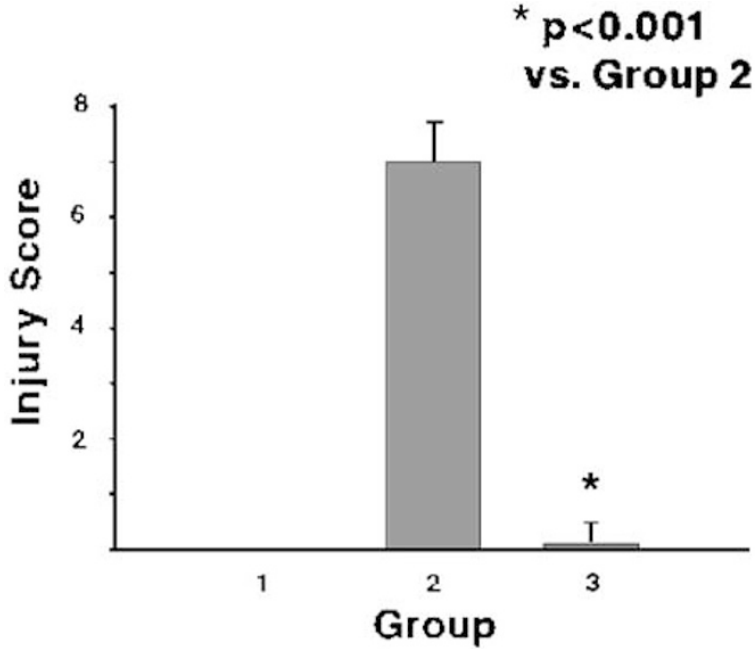

Figure 4 The gross injury score of the stomach from groups 1, 2, and 3 . The injury was almost completely protected by pretreatment of purified ITF in group $3(P<0.001$, ANOVA).

donated by Dr Daniel Podolsky (Boston, MA, USA). This study was approved by the Institutional Ethics Committee of Wenzhou Medical College.

\section{References}

1 Podolsky DK, Lynch-Devaney K, Stow JL, et al. Identification of human intestinal trefoil factor: goblet cell-specific expression of a peptide targeted for apical secretion. J Biol Chem 1993;268:6694-6702.

2 Suemori S, Lynch-Devaney K, Podolsky DK. Identification and characterization of rat intestinal trefoil factor: Tissue- and cell-specific member of the trefoil protein family. Proc Natl Acad Sci 1991;88:11017-11021.

3 Wright NA, Hoffmann W, Otto WR, et al. Rolling in the clover: trefoil factor family (TFF)-domain peptides, cell migration and cancer. FEBS Lett 1997;408:121-123.

4 Mashimo $\mathrm{H}, \mathrm{Wu} \mathrm{DC}$, Podolsky DK, et al. Impaired defense of intestinal mucosa in mice lacking intestinal trefoil factor. Science 1996;274:262-265.

5 Thim L, Woldike HF, Nielsen PF, et al. Characterization of human and rats intestinal trefoil factor produced in yeast. Biochemistry 1995;34:4757-4764.

6 Babyatsky MW, DeBeaumont M, Thim L, et al. Oral trefoil peptides protect against ethanol- and indomethacin-induced gastric injury in rats. Gastroenterology 1996;110:489-497.

7 Lin J, Nadroo AM, Chen W, et al. Ontogeny and prenatal expression of trefoil factor $3 / \mathrm{ITF}$ in the human intestine. Early Hum Dev 2003;71:103-109.

8 Kou RQ, Wang W, Li LY. Chemico-physical properties and spectra of recombinant human intestinal trefoil factor. Chin J Biochem Mol Biol 1999;15:787-791. (in Chinese).

9 Guth PH, Paulsen G, Nacata H. Histologic and microcirculatory changes in alcohol-induced gastric lesions in the rat: effect of prostaglandin cytoprotection. Gastroenterology 1984;87:1083-1090. 